\title{
FUZZY CONTROL OF A SERVOMECHANISM: PRACTICAL APPROACH USING MAMDANI AND TAKAGI-SUGENO CONTROLLERS
}

\author{
Renato Aguiar and Izabella Sirqueira \\ Department of Electrical Engineering, Centro Universitario FEI, S.B.C, Brazil
}

\begin{abstract}
The main objective of this work is to propose two fuzzy controllers: one based on the Mamdani inference method and another controller based on the Takagi-Sugeno inference method, both will be designed for application in a position control system of a servomechanism. Some comparations between the methods mentioned above will be made with regard to the performance of the system in order to identify the advantages of the Takagi-Sugeno method in relation to the Mamdani method in the presence of disturbances and nonlinearities of the system. Some results of simulation and practical application are presented and results obtained showed that controllers based on Takagi-Sugeno method is more efficient than controllers based on Mamdani method for this specific application.
\end{abstract}

\section{KEYWORDS}

Fuzzy Logic, Fuzzy Controller, Mamdani Inference Method, Takagi-Sugeno Inference Method.

\section{INTRODUCTION}

The desire of human beings since first industrial revolution is to gain more control and autonomy of processes in order to obtain more efficiency and productivity. This produces the development of efficient machines, which solve complex problems and perform actions using the same procedure of the reasoning of the human brain through the incorporation of skills to deal with inaccurate and uncertain situations.

In general, industrial processes to contain many nonlinearities and may be affected by external disturbances. These disturbances can damage the performance of the system as a whole and this makes a major challenge with regarding to control of certain variables of a system, so that its dynamics is satisfactory even in presence of disturbances, nonlinearities and uncertainties in the plant model.

However, fuzzy logic is able handle with nonlinearities of a process and can imitate the human procedure in its decisions.

The first publication on fuzzy logic emerged in the context fuzzy sets theory introduced by Lotfi Zadeh in 1965 [1], who created logic combining the concepts of classical logic and Lukasiewicz's sets defining the membership degrees that allow an element partially to belong to more than one cluster simultaneously [2]. In short, fuzzy logic can be defined as a mathematical treatment of certain linguistic terms, such as approximately, very high, very low, around, among others.

Some studies have been done applying these two inference methods: Mamdani and Takagi- 
International Journal of Fuzzy Logic Systems (IJFLS) Vol.11, No.2/3/4, October 2021

Sugeno. As example, in [6] is presented a comparative study of the application of these two methods to predictive the traffic flow. In [7] the authors present a comparative study between Mamdani and Takagi- Sugeno inference methods to control speed of an induction motor drive system. In [8] a comparison between Mamdani and Takagi- Sugeno inference systems is realized for dynamic spectrum allocation. Authors, in [9], applied fuzzy controller for to control position of each finger of a robotic hand and in [10] authors used fuzzy controllers to control vibrations of 3 cars crane system, among others.

In this way, in this work will be used fuzzy set theory to position control of a servomechanism even in presence of disturbances and nonlinearities of the system. More precisely, two inference methods will be used for fuzzy position control: the Mamdani method and the TakagiSugeno method. Simulations of these two fuzzycontrollers applied to the mathematical model of the servomechanism will be realized and an application of these two fuzzy controllers will be realized in the real plant, whererobustness tests will be done.

This paper is organized as follows: in section 2 a review of fuzzy concepts and Mamdaniand Takagi- Sugeno inference methods are presented; in the section 3 the methodology adopted and the results of the simulation and practical application are presented; in section 4 the conclusions are recorded.

\section{THEORY REVIEW}

Fuzzy logic deals with vague or nebulous information, such as: very hot, warm, very high, among others. This theory has skill of handle with imprecise and vagueness situations found in the real world. So, fuzzy logic allows a certain element belong to more than one class simultaneously, but with different membership degrees that are numbers contained in the range [0;1], where the number 0 represents absence of similarity, the number 1 represents total similarities and numbers between 0 and 1 represent partial similarities.

As an example, consider the Figure 1 that represents a set of numbers in the range $[0 ; 10]$. What is the set of numbers approximately equal to 5 ?

Analyzing the Figure 1 it's possible to claim that the number 5 belongs to the set with maximum similarity, because it is the number itself. However, there is no certainty if the number 4.5 belongs to the set of numbers approximately equal to 5 . In this case, the answer is not unique and objective, because belonging or not may depend on the type of problem in question. However, in this case, it can be affirmed that 4.5 belongs to the set of numbers approximately equal to 5 with a degree of similarity approximately equal to 0.9 . 


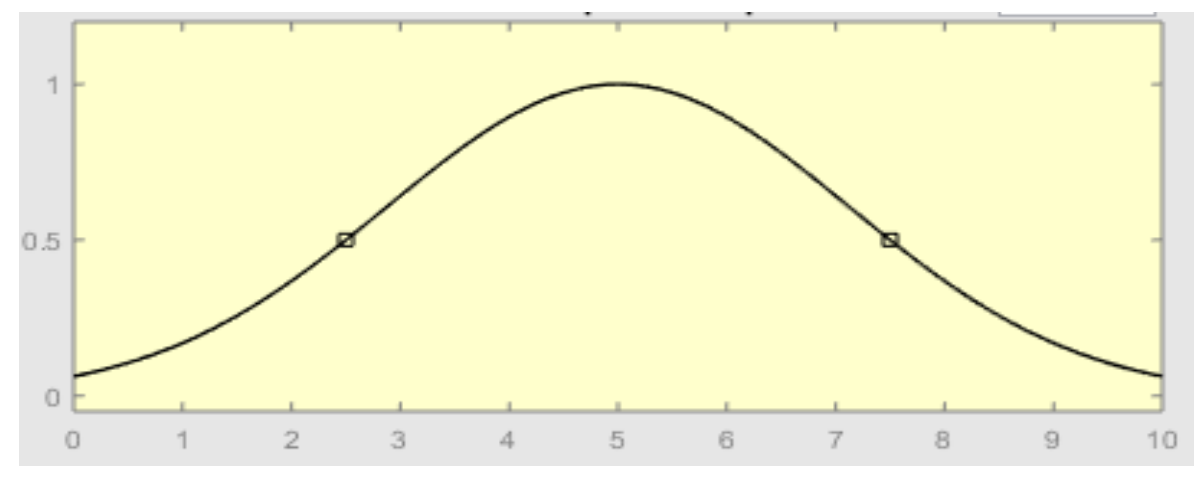

Figure 1. Cluster of the numbers approximately equal to 5

As can be seen, Figure 1 show a gaussian function that consists of numbers close to 5 . Thus, fuzzy logic theory represents a real element in a membership degree with regardto given class. There are numerous functions to represent a class, such as: triangular, gaussian, trapezoidal, among others. These functions are called membership functions and each class is called by a linguistic variable that represents a certain fuzzyness.

Through these functions it is possible to perform some operations with fuzzy sets, such as intersection, union and complement in order to obtain an interrelationship betweenthe system variables. In addition, membership functions are fundamental elements ofthe fuzzy system [3]. Briefly, Figure 2 represents a fuzzy system

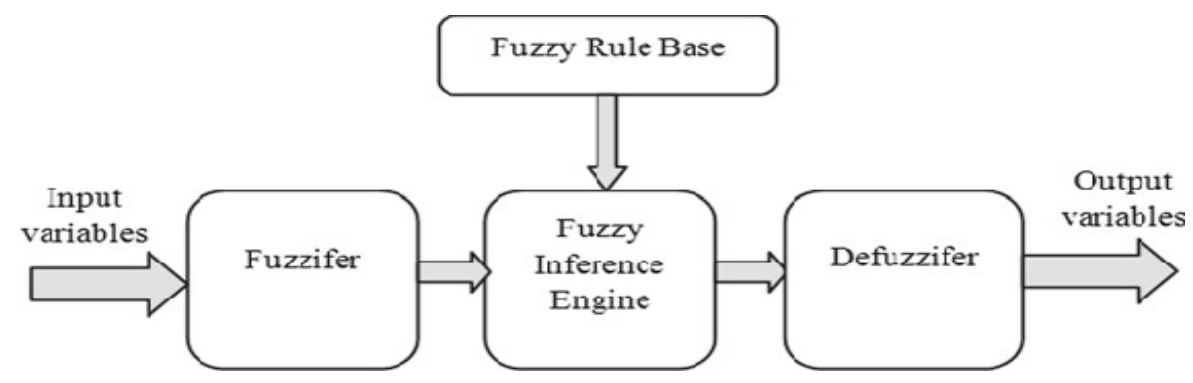

Figure 2. Fuzzy System

a. Input Variables: real values (crisp numbers) such as height equal to 10 meters, temperature equal to 75 degrees Celsius.

b. Fuzzifier: the crisp values are transformed into degrees of similarity.

c. Fuzzy Rule Base: the fuzzy rules, also called "IF-THEN" rules represent the knowledge of the system and information about the parameters of the membership functions (fuzzy sets). The relationship between input and output is produced through of therules.

d. Fuzzy Inference: Performs fuzzy set mapping and interprets input values based on a rule set and assigning a value to the output vector, that is, determining how rules are activated and combined.

e. Defuzzifer: this step converting a fuzzy value into a real number (crisp value). The most used method is based on the calculation of the area centroid of a fuzzy set, where the output value is the center of gravity of the distribution function of possibility of the control action.

f. Output variables: Output in real values.

Clearly, can be noted that the fuzzy controller is a fuzzy system. However, there are two classes of fuzzy controllers very utilized in the practice: the fuzzy controller based on Mamdani's 
inference method [11] and another controller based on Takagi-Sugeno's inference method [12]. The Mamdani method has its rules described according toequation 1 [4].

$$
\text { If } X_{1} \text { is } A_{1} \text { and } X_{2} \text { is } A_{2} \text { then } y=B_{k}
$$

In this case, the consequential is a fuzzy number, defined by a membership function which must go through the defuzzification process. One of defuzzification methods is the center- of- gravity of the means of areas from each rule represented. Equation 2 represents this process [4].

$$
\bar{y}=\frac{\int s y \mu B(y) d y}{\int s \mu B(y) d y}
$$

On the other hand, Takagi- Sugeno's inference method work with rules that follow the format described in equation 3 [5]:

$$
\text { If } X_{1} \text { is } A_{1} \text { and } X_{2} \text { is } A_{2} \text { then } y=g\left(X_{1}, \ldots, X_{m}\right)
$$

As can be seen in equation 9 the consequence is a function of the linguistic variables of the input, and the values from the $\mathrm{y}$ function is weighted by the membership degree from the antecedent, as showed in equation 4 [5].

$$
Y=\frac{\sum_{i=1}^{m} \mu_{A_{i}} \cdot g_{i}}{\sum_{i=1}^{m} \mu_{A_{i}}}
$$

As can be seen, in Takagi- Sugeno's inference method, the defuzzification step does notexist once equation 10 produces a crisp value. In this work, two fuzzy systems (or fuzzycontrollers) will be developed to control the position of a servo mechanism, one based on Mamdani's inference method and another controller based on Takagi- Sugeno's inference method. Some robustness tests will be realized in the practical application in the real plant.

\section{RESUltS}

In this work, the fuzzy controller considered has the configuration shown in Figure 3, where error and derivative of error are the inputs and output is the control effort. 


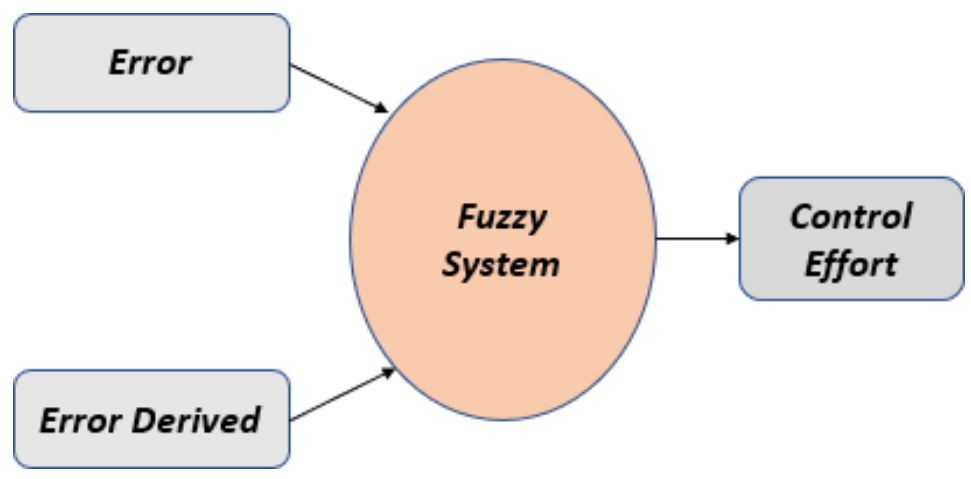

Figure 3. Fuzzy System

The table 1 shows the input and output data, as well as the linguistic values and its respective notation.

Table 1. Variables and linguistic values

\begin{tabular}{|c|c|}
\hline \multicolumn{2}{|c|}{ Linguistic Variable: Error (E) } \\
\hline Linguistic Value & Notation \\
\hline very negative & $\mathrm{VN}$ \\
\hline negative & $\mathrm{N}$ \\
\hline null & $\mathrm{NN}$ \\
\hline positive & $\mathrm{P}$ \\
\hline very positive & $\mathrm{VP}$ \\
\hline
\end{tabular}

Linguistic Variable: Derivative of Error (DE)

\begin{tabular}{|c|c|}
\hline Linguistic Value & Notation \\
\hline Small & $\mathrm{S}$ \\
\hline Large & $\mathrm{L}$ \\
\hline \multicolumn{2}{|c|}{ Linguistic Variable: Control Effort (CE) } \\
\hline Linguistic Value & Notation \\
\hline Very Negative & $\mathrm{VN}$ \\
\hline Negative Medium & $\mathrm{NM}$ \\
\hline Medium & $\mathrm{M}$ \\
\hline Positive Medium & $\mathrm{PM}$ \\
\hline Very Positive & VP \\
\hline
\end{tabular}

The Figure 4 shows the block diagram utilized in simulation, where the block called dead zone is representing the friction existing in the motor. 
International Journal of Fuzzy Logic Systems (IJFLS) Vol.11, No.2/3/4, October 2021

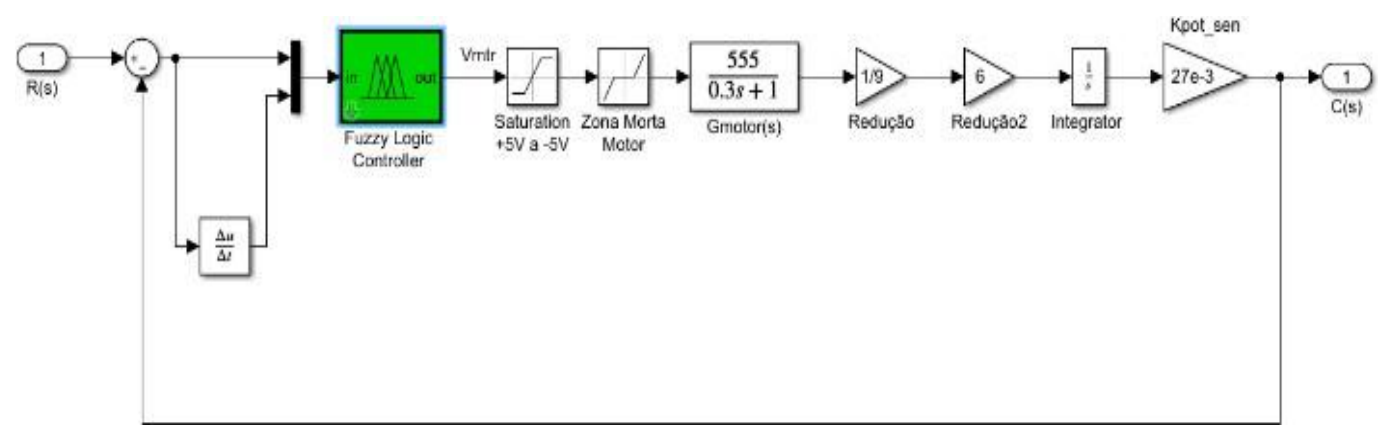

Figure 4. Fuzzy Control System for the simulation

Figure 4 represents a position control system, and the work consists of two steps: in order to assessing and validating the proposed controllers, simulation is done using the Matlab software. After, the fuzzy controllers are applied in the real plant where some robustness tests will be done in the presence uncertainties and disturbances. The table 2 summarizes the rules set proposed when Mamdani inference method is applied.

Table 2. Mamdani rules

\begin{tabular}{|c|c|c|l|c|c|}
\hline & NVL & NL & N & PL & PVL \\
\hline S & X & X & PM & X & X \\
\hline L & X & X & NM & X & X \\
\hline X & VN & NM & M & PM & VP \\
\hline
\end{tabular}

In table 2, $\mathrm{X}$ means not significant, that is, it does not significantly interfere in the dynamics of the system. The membership functions utilized were obtained after many tests and are shown in figures 5, 6 and 7.

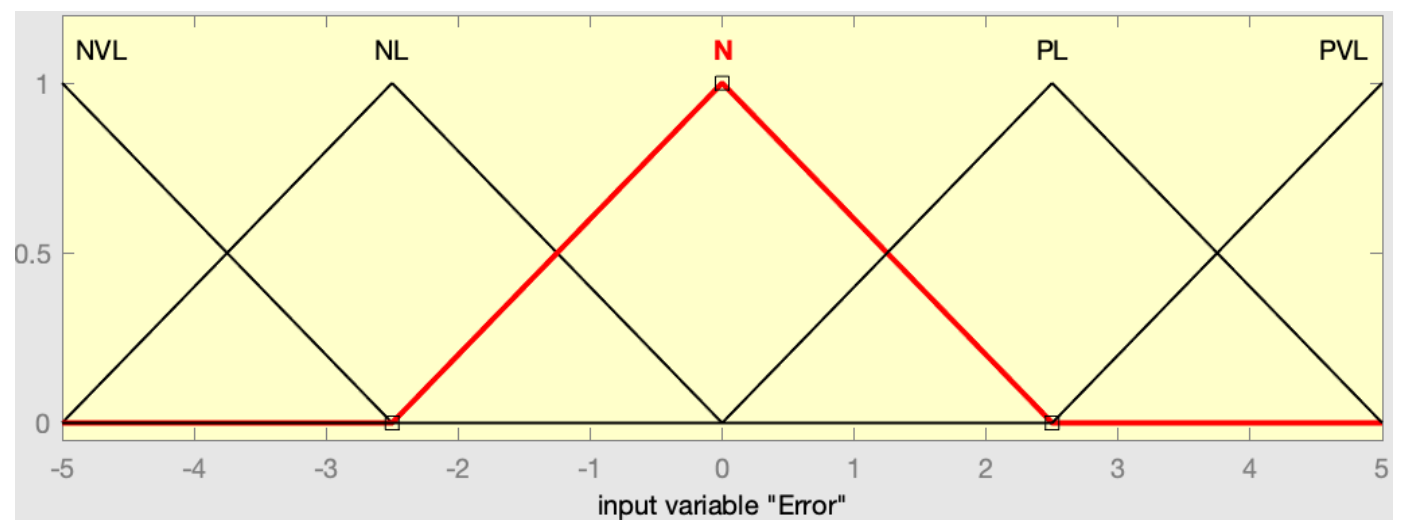

Figure 5. Membership functions: Error 
International Journal of Fuzzy Logic Systems (IJFLS) Vol.11, No.2/3/4, October 2021

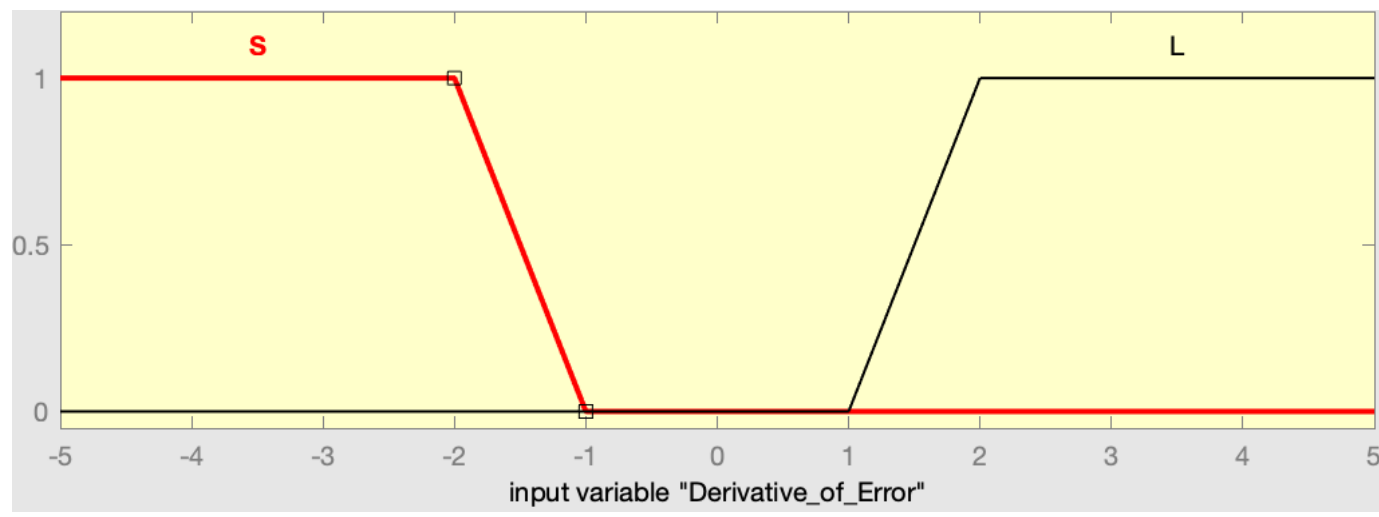

Figure 6. Membership functions: Derivative of error

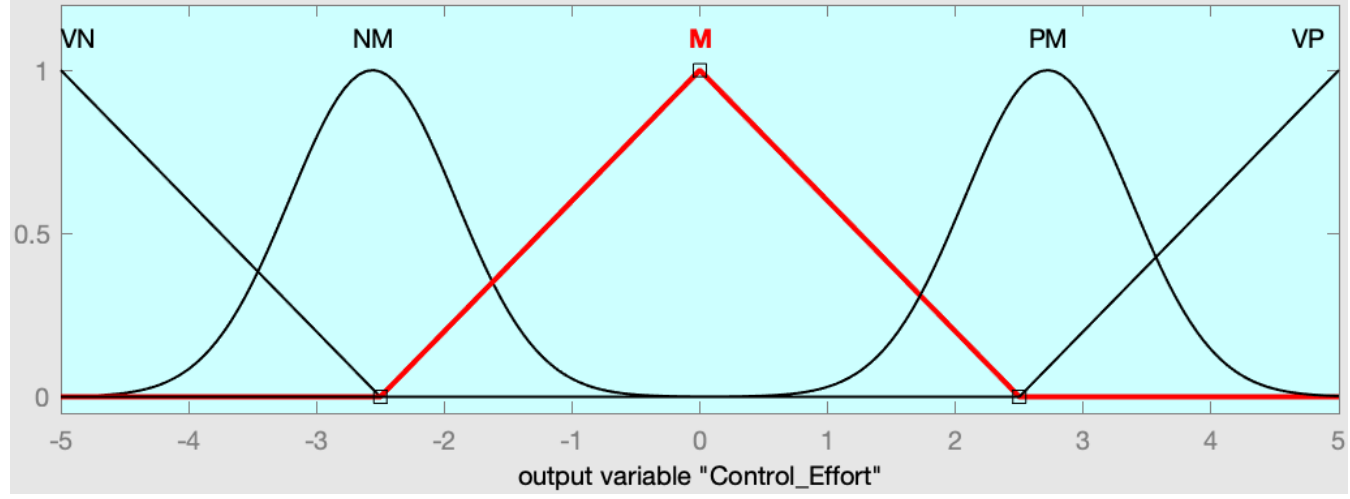

Figure 7. Membership functions: Output

The rules defined in Takagi- Sugeno inference method is shown in table 3.

Table 3. Takagi-Sugeno rules

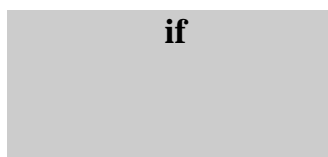

Error is null

Error is negative
Error is positive
$\begin{gathered}\text { Error is positive } \\ \text { medium }\end{gathered}$

Error is negative medium

Error is null

Error is null
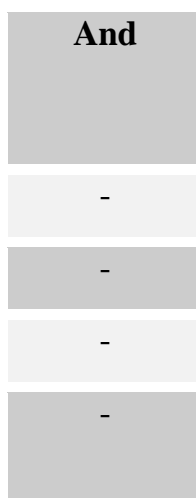

$$
\quad Y=x_{1}-2
$$

Derivativeof $\quad Y=x_{2}$ error

Derivativeof $\quad Y=-x_{2}$ error
Then control effort is

0

$-5$

5

$Y=x_{1}+2$

$Y=x_{X}$ 


$$
\text { where } x_{1}=\text { error and } x_{2}=\text { derivative of error }
$$

These rules were obtained through of knowledge of plant after many tests and changing rules and membership functions until to obtain a satisfactory response.

\subsection{Simulation Results}

The simulations were done through of Matlab software and considering mathematical model of the plant as shown in Figure 4. The system response to the step as a set point and applying Mamdani's inference method is shown in Figure 8.

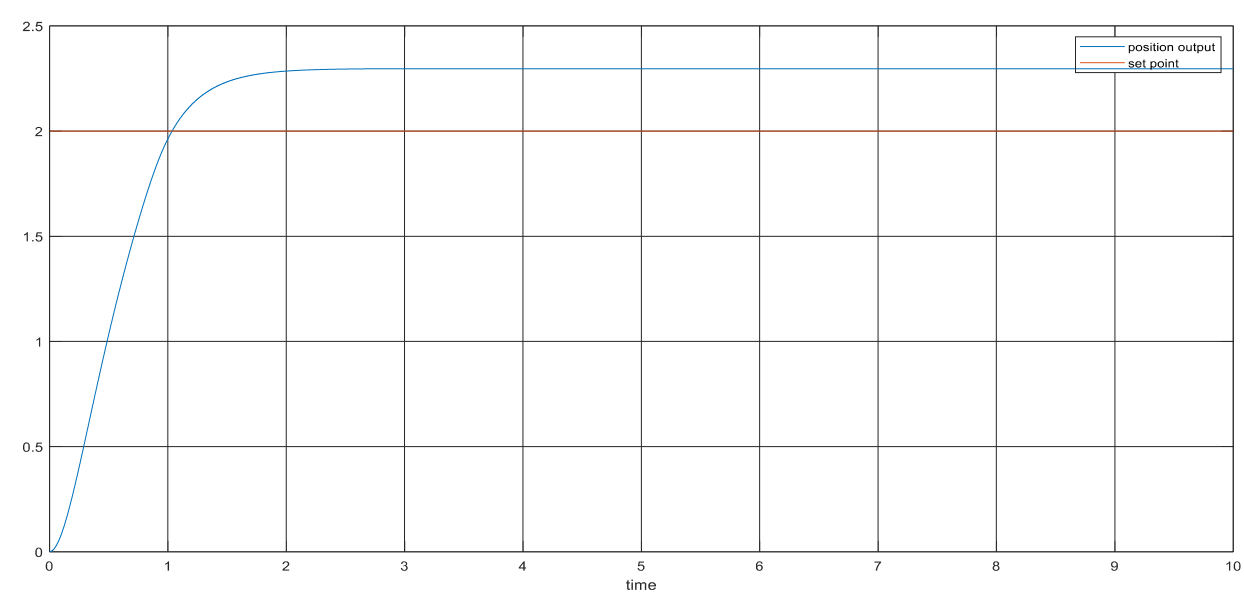

Figure 8. Response to the step of value equal to 2- Mamdani

Figure 9 shows the output of the system when is applied Takagi- Sugeno's inference method.

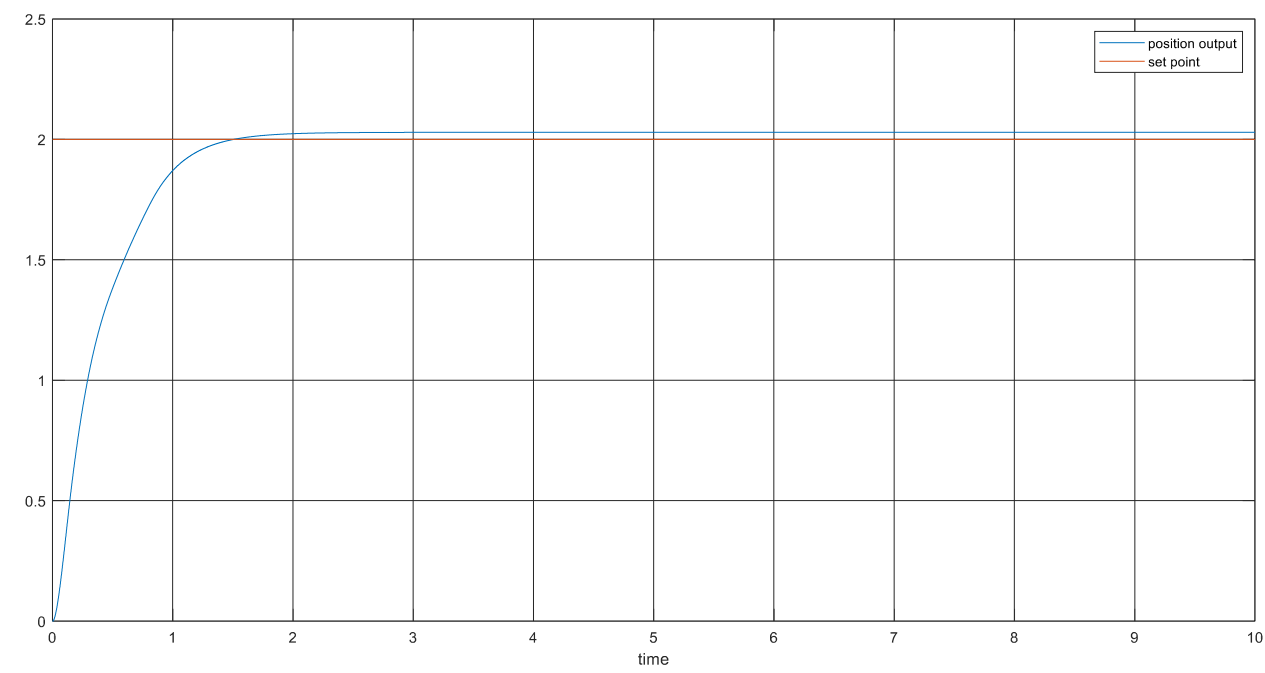

Figure 9. Response to the step of value equal to 2- Takagi- Sugeno

As can be seen in figures 8 and 9, the Takagi- Sugeno inference method presents better results than Mamdani inference with regard to the steady state error and settling time. Table 4 show the approximated values of performance of the system. 
Table 4. Comparison of Performance- Simulation

\begin{tabular}{|c|c|c|}
\hline \multicolumn{2}{|l|}{ Output Performance } \\
\hline & Mamdani & Takagi- Sugeno \\
\hline Error & 0.3 & 0.07 \\
\hline Settling Time & 2 seconds & 1.5 second \\
\hline Overshoot & $0 \%$ & $0 \%$ \\
\hline
\end{tabular}

This application in simulation shows that the fuzzy controller proposed can be promising in application in a real plant. It is known that mathematical model is an approximation of the real plant and do not consider non- linearities, backlash effect and some parameters of the system when compared to the real plant. So, becomes necessary to apply the fuzzy controllers obtained in the real plant which contain non- linearities, backlash effect due to the gears and some noises.

\subsection{Experimental Results}

The fuzzy controllers shown in the preview section will be implemented in a real systemthrough data acquisition process using the data acquisition toolbox contained in Matlab software. Here was utilized a real servomechanism, a data acquisition board National Instruments 6221- 37 pin installed in the computer and an interface National Instruments for communication between Simulink and real plant. The Figure 9 illustrates a basic idea of the configuration utilized.

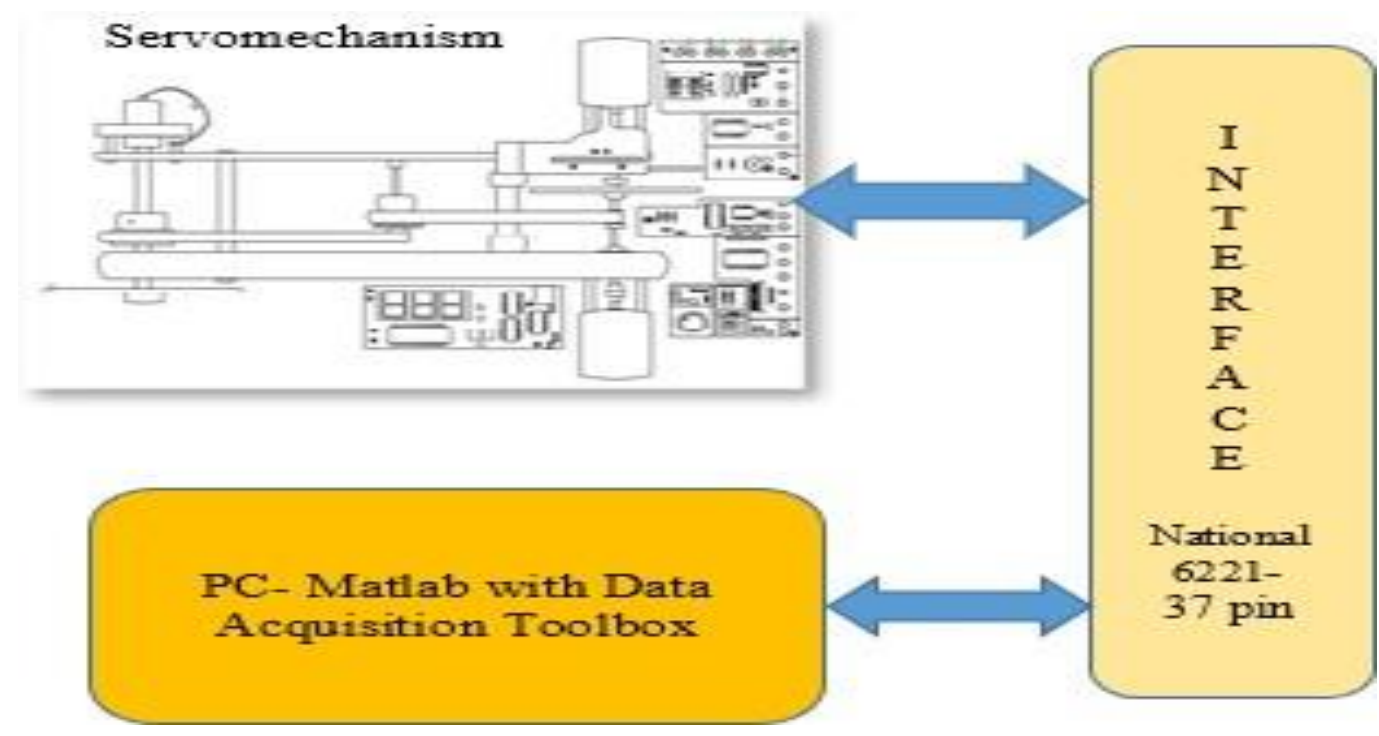

Figure 9. Layout for tests in real plant

Figure 10 shows the model utilized in the Simulink/ Matlab using data acquisition. The set point is a square wave form of $0.2 \mathrm{~Hz}$ and peak value equal to 2 . 
International Journal of Fuzzy Logic Systems (IJFLS) Vol.11, No.2/3/4, October 2021

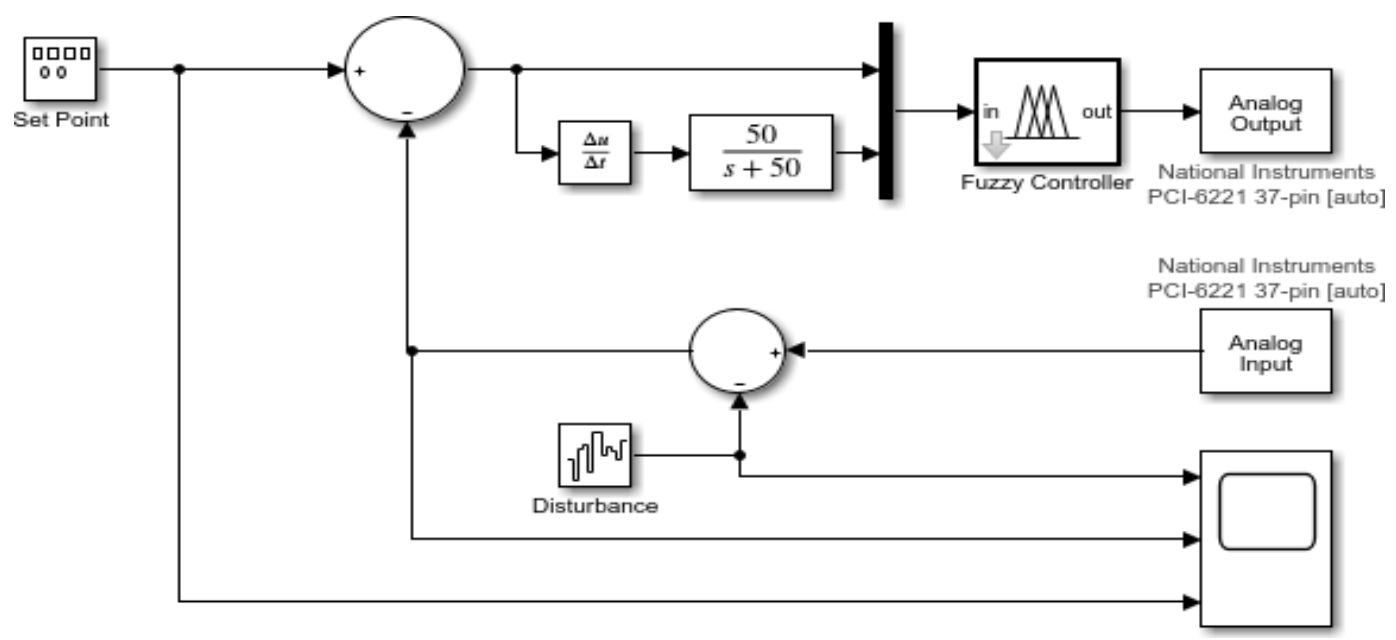

Figure 10. Fuzzy Control System

The membership functions of the inputs and outputs are showed in figures 11, 12 and 13, respectively. In this practical application was necessary to change membership functions.

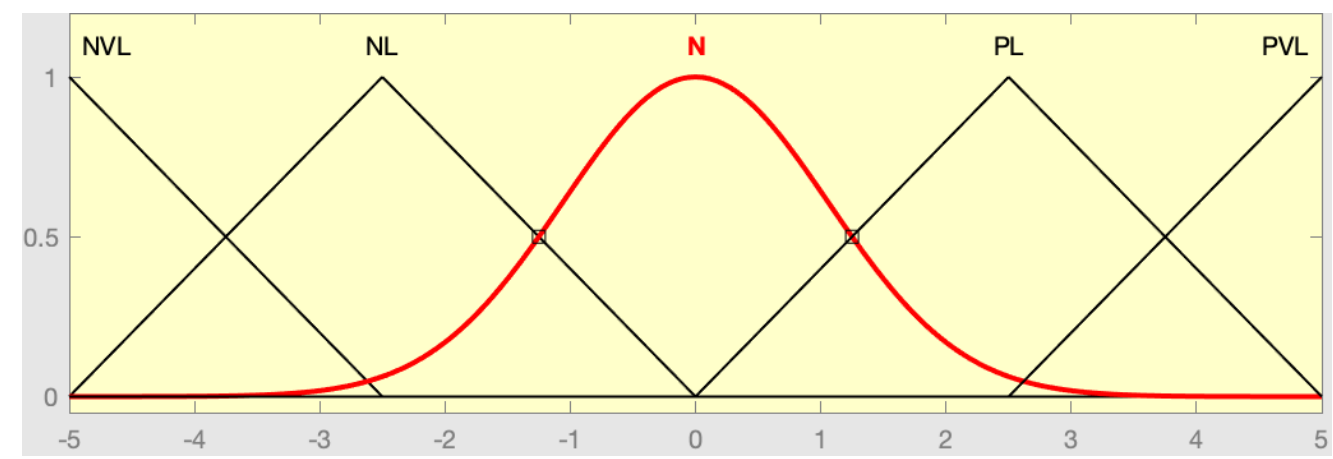

Figure 11. New membership functions: Error

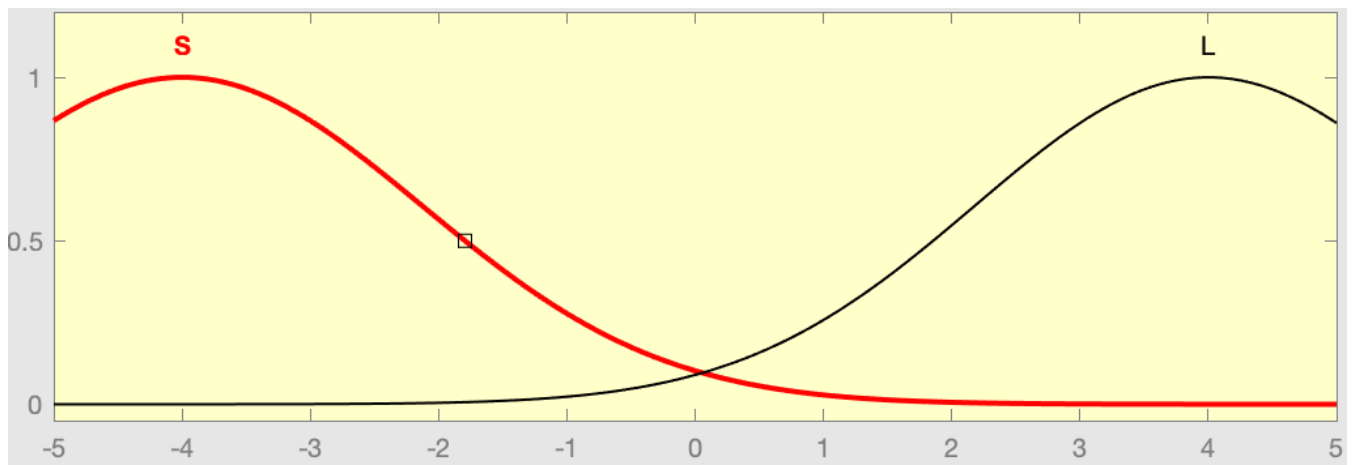

Figure 12. New membership functions: Derivative of error 
International Journal of Fuzzy Logic Systems (IJFLS) Vol.11, No.2/3/4, October 2021

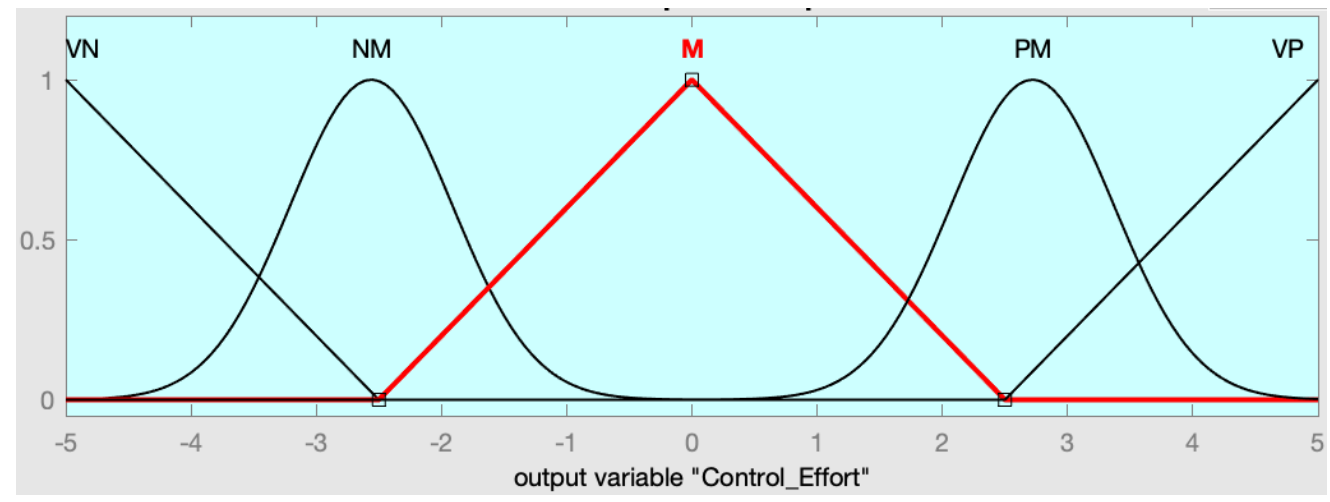

Figure 13. New membership functions: Output

The rules adopted are shown in table 5 .

Table 5. Mamdani rules

\begin{tabular}{|c|c|c|}
\hline if & And & Then control effort is \\
\hline Error is null & - & null \\
\hline $\begin{array}{l}\text { Error } \\
\text { negative }\end{array}$ & - & negative \\
\hline Error is positive & - & positive \\
\hline $\begin{array}{l}\text { Error is positive } \\
\text { medium }\end{array}$ & - & positive medium \\
\hline $\begin{array}{l}\text { Error is } \\
\text { negativemedium }\end{array}$ & - & negative medium \\
\hline Error is null & $\begin{array}{l}\text { Derivative of } \\
\text { error }\end{array}$ & positive medium \\
\hline Error is null & $\begin{array}{l}\text { Derivative of } \\
\text { error }\end{array}$ & negative medium \\
\hline
\end{tabular}

The figures 14 and 15 show the results obtained without disturbance and with disturbance, respectively. 
International Journal of Fuzzy Logic Systems (IJFLS) Vol.11, No.2/3/4, October 2021

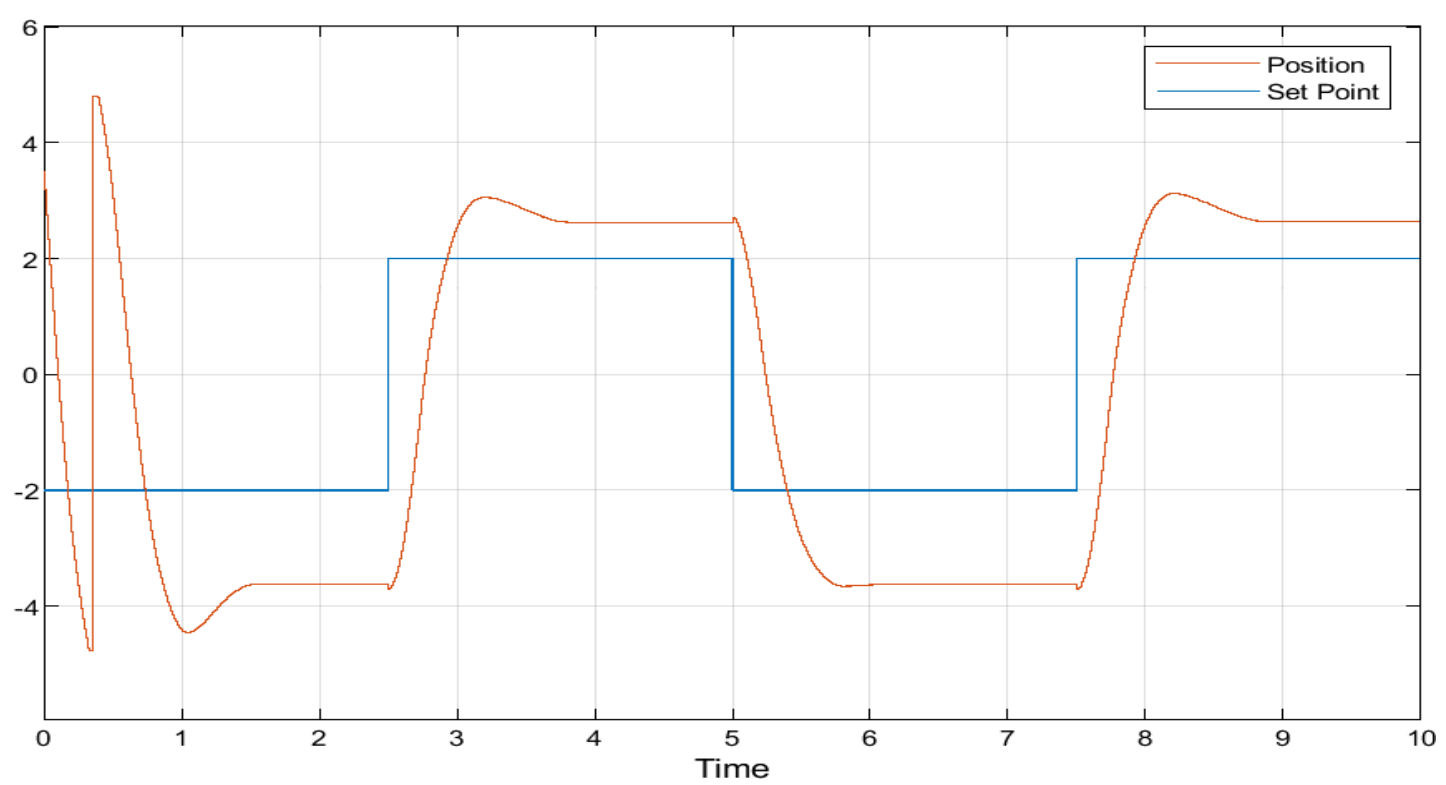

Figure 14. Response of the real system using the Mamdani inference method without disturbance

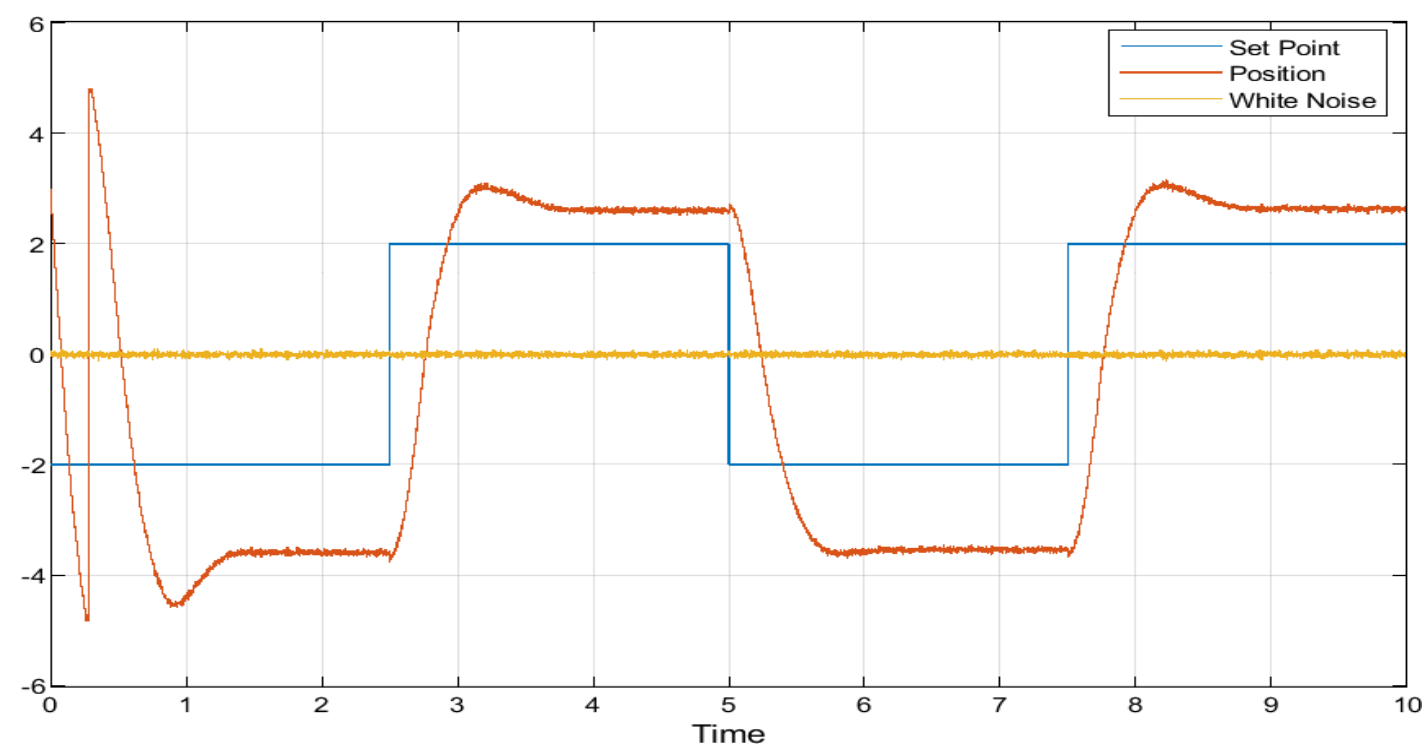

Figure 15. Response of the real system using the Mamdani inference method with the addition ofdisturbance

The same procedure was realized applying the Takagi- Sugeno's inference method. Inthis case, the rules that produced the best results are shown in table 6 . 
International Journal of Fuzzy Logic Systems (IJFLS) Vol.11, No.2/3/4, October 2021

Table 6. Takagi-Sugeno rules

\begin{tabular}{|c|c|c|}
\hline if & And & Then control effort is \\
\hline Error is null & & 0 \\
\hline $\begin{array}{l}\text { Error } \\
\text { negative }\end{array}$ & & -3 \\
\hline Error is positive & & 3 \\
\hline Error is positive & & $Y=x_{1}+2$ \\
\hline \multicolumn{3}{|l|}{ medium } \\
\hline $\begin{array}{l}\text { Error is } \\
\text { negativemedium }\end{array}$ & - & $Y=x_{1}-2$ \\
\hline Error is null & Derivative of error & $Y=3 x_{1}+6 x_{2}$ \\
\hline Error is null & Derivative of error & $Y=3 x_{1}+6 x_{2}$ \\
\hline
\end{tabular}

The figures 16 and 17 shown the response of the system without disturbance and with disturbance, respectively.

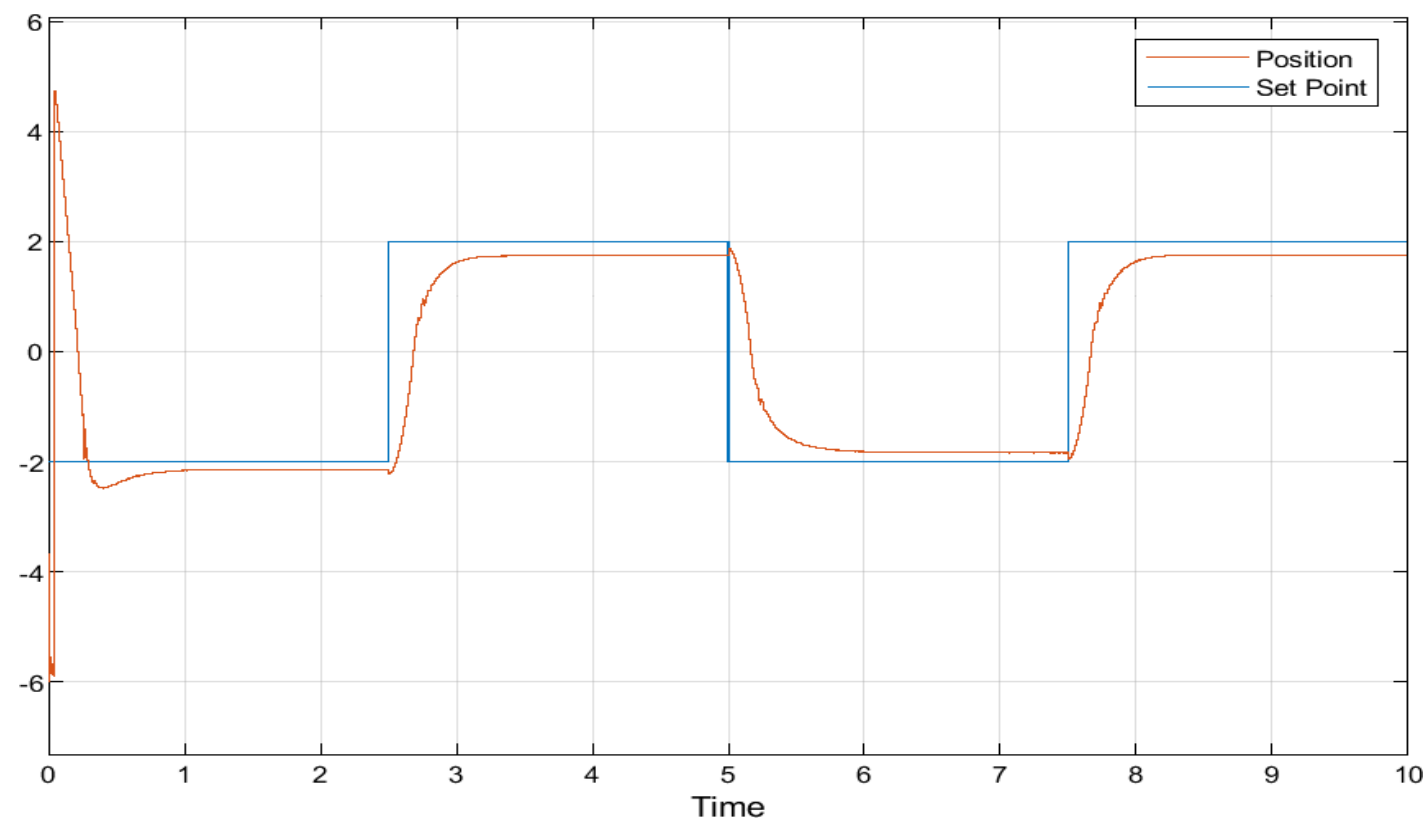

Figure 16. Response of the real system using the Takagi-Sugeno inference method without disturbance 


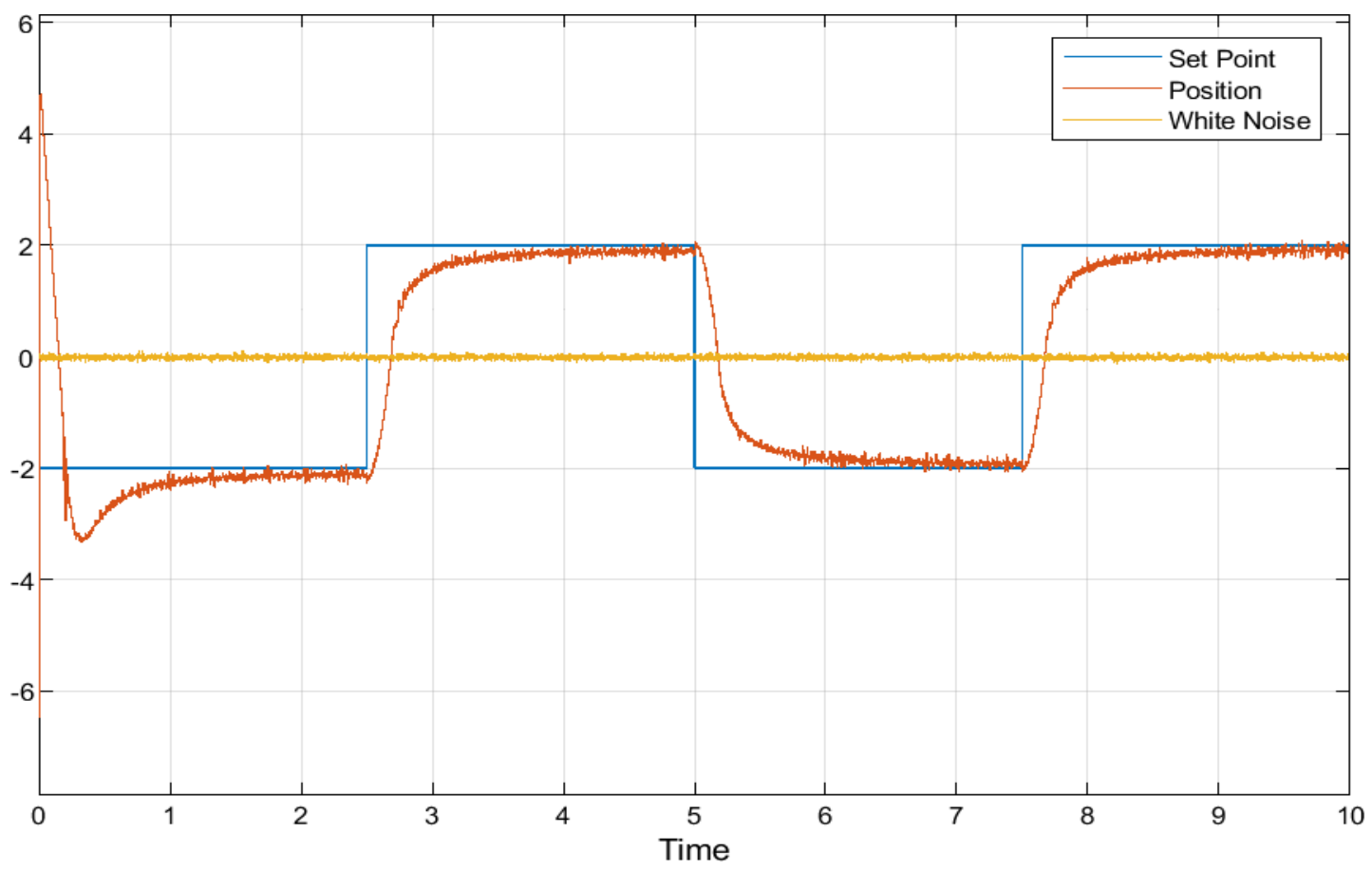

Figure 17. Response of the real system using the Takagi-Sugeno inference method with theaddition of disturbance

As can be seen in the experimental results presented, Takagi- Sugeno inference method presents a better result than Mamdani inference method in the application proposed in this work. As can be seen in figures 16 and 17, when is utilized the fuzzy controller based in the Takagi- Sugeno inference method, the response of the system is faster than fuzzy controller based in the Mamdani inference method. Table 7 show the approximated values of performance of the system

Table 7. Comparison of Performance- Simulation

\begin{tabular}{|c|c|c|}
\hline \multicolumn{2}{|l|}{ Output Performance } \\
\hline & Mamdani & Takagi- Sugeno \\
\hline Error & 0.56 & 0.22 \\
\hline Settling Time & 1.5 seconds & 0.5 second \\
\hline Overshoot & $3 \%$ & $0 \%$ \\
\hline
\end{tabular}

Furthermore, the results indicate that for the application proposed here, the controller based on Takagi- Sugeno inference method seems has more robustness and precision when compared to the Mamdani inference method.

\section{Conclusions}

In this work, was proposed a position control using a fuzzy controller. The applicationis based in two controllers: a fuzzy controller using Mamdani inference method and another controller using Takagi- Sugeno inference method. Firstly, was done a simulation using the 
mathematical model of a servomechanism in order to assessing and validating the proposed controllers. After, the two controllers were applied in real plant, where nonlinearities, noises and backlash effect are present in the system.

Analyzing the responses presented by the simulations, Takagi-Sugeno inference methodpresents low settling time and very low steady state error. When comparing the results obtained with the experiments in real plants, was found that the method of inference Takagi-Sugeno presents greater robustness, less steady state error and less settling time even in the presence of disturbance and nonlinearities of the system. For both methods, changes in the membership functions were necessary in practical application due to some non-linearities, noises and backlash effect.

So, in the application proposed in this work, Takagi- Sugeno inference method toposition control in the presence of uncertainties, disturbance and nonlinearities, produces a better result when compared with Mamdani inference method Here, the control based on Takagi- Sugeno inference method shown more robustness and better relative stability. As future study, can be very promising the application of control basedon fuzzy type- 2 .

\section{REFERENCES}

[1] Zadeh L. A. Fuzzy sets, Information and Control, p. 338-353, 1965.

[2] Zimmermann H. J. Fuzzy Set Theory and its Aplication. Kluwer Academic, Boston, 1996.

[3] Altrok, C.V. Fuzzy Logic \& Neurofuzzy Applications Explained, Prentice Hall,New Jersey, 1995.

[4] Mamdani E. H; Assilian S. An Experiment in Linguistic Synthesis with a Fuzzy Logic Controller. Int. J. Man- Machine Studies, v.7, p. 1-13, 1975.

[5] Takagi T., Sugeno M. Fuzzy Identification of Systems and Its Applications to Modeling and Control. IEEE Transactions on Systems, Man, and Cybernetics, v.15, 1985.

[6] Wang Y., Chen, Y. A Comparison of Mamdani and Sugeno Fuzzy InferenceSystems for Traffic Flow Prediction. Journal of Computers, v.9, n. 1, 2014.

[7] Ariff, R.M.; Hanafi, D.; Utomo, W.M.; Sim, S.Y.; Bohari, A.A. Comparison of Sugeno-Type and Mamdani-Type Speed Controller for Induction Motor Drive System. In Proceedings of the 3rd International Conference on Computer Engineering and Mathematical Sciences (ICCEMS 2014), Langkawi, Malaysia,4-5 December 2014; pp. 387-391.

[8] Subhedar M, Birajdar G. Comparison of Mamdani and Sugeno inference systems for dynamic spectrum allocation in cognitive radio networks. Wireless Pers Commun.; v. 71, p.805-819, 2013.

[9] Rakovic M. et al. Fuzzy Position- velocity Control of Underactuated Finger of FTN Robot Hand. Journal of Intelligent and Fuzzy Systems, v. 34, 2018.

[10] Yildirim S. Angular Position Control of 3- Cars Crane System Using ProposedFuzzy- PID Controller. Recent Innovations in Mechatronics, v.7, 2020.

[11] Mamdani E. H. Application of Fuzzy Logic to Approximate Reasoning Using Linguistic Synthesis. IEEE Transactions on Computers, v. C26, p. 1182- 1191,1977.

[12] Takagi T., Sugeno M. Fuzzy Identification of Systems and its Applications to Modeling and Control. IEEE Transactions on Systems, Man, and Cybernetics, v.SMC-15, p. 116- 132, 1985. 